\title{
Contents and Uptake Rates of Mn, Fe, Co, Zn, and Se in Se-Deficient Rat Liver Cell Fractions
}

\author{
Ken-ichiro Matsumoto, ${ }^{* \dagger}$ Tetsuya Inagaki, ${ }^{*}$ Rieko Hirunuma, ${ }^{* *}$ Shuichi Enomoto, ${ }^{* *}$ \\ and Kazutoyo ENDO* \\ * Department of Physical Chemistry, Showa Pharmaceutical University, \\ 3-3165 Higashi-Tamagawagakuen, Machida, Tokyo 194-8543, Japan \\ **Radioisotope Technology Division, Cyclotron Center, RIKEN (The Institute of Physical and Chemical Research), 2 - \\ 1 Hirosawa, Wako, Saitama 351-0198, Japan
}

\begin{abstract}
The contents of manganese $(\mathrm{Mn})$, iron $(\mathrm{Fe})$, cobalt $(\mathrm{Co})$, zinc $(\mathrm{Zn})$, and selenium $(\mathrm{Se})$ in nuclear $(\mathrm{NU})$, mitochondrial (MT), microsomal (MC), and cytosolic (CS) fractions of liver homogenates of normal and selenium-deficient (SeD) rats were determined by instrumental neutron activation analysis (INAA). The uptake rates of these elements in the liver cell fractions of both groups of rats were determined by multitracer analysis (MTA). The results indicated that Se-deficiency caused a significant increase in the content of Fe in the MC fractions. The MTA showed that the uptake rate of Fe was highest in the MC fraction, and that the uptake rate in the fraction was similar between the SeD and normal rats.
\end{abstract}

(Received November 5, 2000; Accepted February 27, 2001)

\section{Introduction}

Selenium (Se) is an essential trace element that constitutes the active center of glutathione peroxidase (GSH-Px). ${ }^{1,2}$ GSH-Px reduces hydrogen peroxide $\left(\mathrm{H}_{2} \mathrm{O}_{2}\right)$ to water using a reduced form glutathione $(\mathrm{GSH})$. It is therefore thought that $\mathrm{Se}$ interrupts the formation of the hydroxyl radical $(\cdot \mathrm{OH})$ from $\mathrm{H}_{2} \mathrm{O}_{2}$ through Harber-Weiss and/or Fenton reactions. ${ }^{3,4}$ In other words, Se plays numerous protective roles against oxidative injury, including the peroxidation of membrane lipids and the degeneration of DNA and/or proteins.

On the other hand, it is known that Se interacts with other metals in biological systems. For example, previous papers have reported an enhanced accumulation of iron $(\mathrm{Fe})$ in the liver, kidney, and spleen of Se-deficient $(\mathrm{SeD})$ rats.5,6 The pathological phenomena of Se-deficiency are very similar to those caused by an Fe-overload. ${ }^{7}$ Inversely, it has also been reported that Fe-deficiency causes a decrease of GSH-Px activities in red blood cells and liver. ${ }^{8,9}$ However, the mechanisms of $\mathrm{Fe}$ accumulation and the function of the accumulated $\mathrm{Fe}$ in $\mathrm{SeD}$ rats have not yet been clarified. In living organisms, $\mathrm{Fe}$ constitutes the active center of catalase, cytochrome P-450 (P-450), and xanthine oxidase, and is related to the digestion and synthesis of reactive oxygen species (ROS). Not only Fe, but also other metal ions, are present in antioxidant enzymes and/or vitamins, and collaborate with each other in complex ways to maintain the valance in in vivo redox systems. ${ }^{10-12}$ A recent study showed that the in vivo total reducing capacity decreased in the liver and head of SeD rats. ${ }^{13}$

† To whom correspondence should be addressed.

E-mail: knmatsu@ac.shoyaku.ac.jp

T. I. present address: Department of Pharmacy, KokusaiShinzen Sougou Hospital, 1-28-1 Nishigaoka, Izumi, Yokohama, Kanagawa 245-0006, Japan.
Therefore, the change in the in vivo redox valance due to decreased GSH-Px activity is expected to change the dynamics of the metal elements in the $\mathrm{SeD}$ rat. Although the contents of the metal elements in various rat organs have been reported, the detailed distributions of elements related to the redox reactions have not yet been studied.

In this experiment, we used instrumental neutron activation analysis (INAA) and multitracer analysis (MTA) ${ }^{14,15}$ to examine the contents and uptake rates of Se, manganese (Mn), Fe, cobalt (Co), and zinc ( $\mathrm{Zn})$ in the liver cell fractions of SeD rats. The results were compared with those found for normal rats, and discussed in relation to oxidative stress caused by $\mathrm{Se}$ deficiency.

\section{Experimental}

\section{Materials}

NADPH, GSH, and GSH reductase were purchased from Sigma Chemical Co. (St. Louis, MO). All other materials used were of analytical grade. The normal diet (CE-2) was purchased from CLEA Japan, Inc. (Tokyo, Japan). The SeD diet was purchased from Oriental Yeast Co., Ltd. (Tokyo, Japan). The SeD diet contains $0.008 \mathrm{mg} / \mathrm{kg}$ selenium estimated with fluorometric analysis. ${ }^{16}$ Deionized water (deionization by the Milli-Q system) was used as ultra pure water.

\section{Animals}

Rats were purchased from Japan Laboratory Animals, Inc. (Tokyo, Japan). Mother Wistar rats (15th days of pregnancy) were fed on the SeD diet and ultra-pure water until weaning of their babies. Four-week-old male rats were weaned and fed the SeD diet and ultra-pure water until the experiments. These rats comprised the SeD rat model group $(110 \pm 25 \mathrm{~g}$ at 8 weeks of age, $n=35$ ). Normal male Wistar rats of the same age as the $\mathrm{SeD}$ rats were used as a control group $(224 \pm 16 \mathrm{~g}$ at 8 weeks of 
age, $n=37$ ).

Contents of $\mathrm{Se}, \mathrm{Mn}, \mathrm{Fe}, \mathrm{Co}$, and $\mathrm{Zn}$ in the diet, liver and liver cell fractions

The normal and the SeD diet were lyophilized and ground into a powder. Three normal and $5 \mathrm{SeD}$ rats (7 weeks of age) were sacrificed by decapitation. Blood was taken from the cut as much as possible. The livers were removed, lyophilized, and ground into a powder. In the liver samples, a small amount of blood remained. For the sample of liver cell fractions, six normal and $6 \mathrm{SeD}$ rats ( 8 weeks of age) were anesthetized with $0.05 \mathrm{mg} / \mathrm{g}$ b.w. intraperitoneal injection of Nembutal (Dinabot, Osaka, Japan) and then sacrificed by bleeding from the abdominal aorta. Subsequently, the liver was perfused with icecold physiological saline $(0.9 \% \mathrm{NaCl})$ until the blood was sufficiently removed. The liver was then removed and homogenized with a 2-fold volume of $1.15 \% \mathrm{KCl}$. Finally, nuclear (NU), mitochondrial (MT), microsomal (MC), and cytosolic (CS) fractions were separated by centrifugation. Each fraction was lyophilized and ground into a powder. An aliquot of each powdered sample was weighed and sealed in polyethylene. The Standard Reference Material 1577 (bovine liver) obtained from the National Bureau of Standards (Gaithersburg, MD) was used to check the analyses of inorganic elements.

Neutron irradiation was carried out in a TRIGA II nuclear reactor at Rikkyo University. The $\gamma$-ray spectra of the irradiated samples were measured with a high-purity Ge semiconductor detector equipped with a multichannel analyzer (Seiko EG \& G Co., Ltd., Matsudo, Japan). ${ }^{75} \mathrm{Se},{ }^{56} \mathrm{Mn},{ }^{59} \mathrm{Fe},{ }^{60} \mathrm{Co}$, and ${ }^{65} \mathrm{Zn}$ were analyzed by the photopeaks at 264.7, 846.8, 1099.2, 1332.4 , and $1115.5 \mathrm{keV}$, respectively. The peak identification of $\gamma$-ray spectra was based on a criterion of above $3 \sigma$ (threetimes the standard deviation of the background counts).

Uptake rates of $\mathrm{Se}, \mathrm{Mn}, \mathrm{Fe}, \mathrm{Co}$, and $\mathrm{Zn}$ in the liver cell fractions A multitracer hydrochloric acid solution $(2 \mathrm{~mL})$ was prepared from a silver foil irradiated with a ${ }^{14} \mathrm{~N}$ beam of 135 MeV/nucleon at the RIKEN Ring Cyclotron. ${ }^{14,15}$ Three 8-weekold normal and SeD rats were anesthetized with diethylether. Then, a $100 \mu \mathrm{L}$ volume of multitracer solution was administered (i.v.) to each rat. Two days later, the NU, MT, MC, and CS fractions were prepared from each rat liver by centrifugation. An aliquot $(1.0 \mathrm{~g})$ of each wet fraction was put into a plastic tube. As the standard sample, $1.0 \mathrm{ml}$ of a diluted (1/100) multitracer solution was put into a plastic tube of the same size. The measurement and analysis of the $\gamma$-ray spectra were performed by the same procedure as INAA. In the MTA, ${ }^{75} \mathrm{Se}$, ${ }^{54} \mathrm{Mn},{ }^{59} \mathrm{Fe},{ }^{56} \mathrm{Co}$, and ${ }^{65} \mathrm{Zn}$ were analyzed by the photopeaks at 264.7, 834.8, 1099.2, 810.8, and $1115.5 \mathrm{keV}$, respectively. Taking into account the difference in the total body weight between the normal and $\mathrm{SeD}$ rats, the uptake rate was normalized as a percentage of the photopeak counts per $1 \mathrm{~g}$ cell fraction to those in the standard multitracer solution per total body weight: ${ }^{17}$

$$
\text { Uptake rate }(\%)=\frac{\text { Radioactivity in } 1 \mathrm{~g} \text { cell fraction }}{\begin{array}{l}
\text { Radioactivity in administered tracer } \\
\text { solution/Body weight }
\end{array}} \times 100 \text {. }
$$

The $100 \%$ uptake rate indicates a uniform distribution of each nuclide in the whole body.

Cytosolic GSH-Px activity and thiobarbituric acid reactive substance (TBARS) level

Five normal and $5 \mathrm{SeD}$ rats (8 weeks of age) were used for the liver cytosolic GSH-Px assay and the TBARS assay. After the
Table 1 Contents of bio-trace elements in the diet and the rat liver

\begin{tabular}{ccccc}
\hline Element & $\begin{array}{c}\text { Normal } \\
\text { diet }\end{array}$ & SeD diet & $\begin{array}{c}\text { Normal } \\
\text { rat liver }\end{array}$ & SeD rat liver \\
\hline $\mathrm{Se}$ & $0.86 \pm 0.13$ & N.D. & $3.2 \pm 0.2$ & N.D. \\
$\mathrm{Mn}$ & $516 \pm 25$ & $105 \pm 4.4^{* *}$ & $8.4 \pm 0.8$ & $6.3 \pm 0.6^{*}$ \\
$\mathrm{Fe}$ & $482 \pm 5.6$ & $583 \pm 7.6^{* *}$ & $525 \pm 49$ & $1326 \pm 108^{* *}$ \\
$\mathrm{Co}$ & $0.9 \pm 0.02$ & $0.03 \pm 0.002^{* *}$ & $0.8 \pm 0.1$ & $0.04 \pm 0.01^{*}$ \\
$\mathrm{Zn}$ & $62 \pm 1.8$ & $60 \pm 0.9$ & $100 \pm 4.2$ & $112 \pm 4.3 \#$ \\
\hline
\end{tabular}

The values were obtained as $\mathrm{mg} / \mathrm{kg}$ in dried powder sample and shown as means S.D. of three different measurements. Three normal and $5 \mathrm{SeD}$ Wistar male rats (7 weeks of age) were used for preparation of the liver samples. N.D.: not detected. \#, *, and ** indicate significant difference between the normal and the $\mathrm{SeD}$ diet/rats at $p<0.05, p<0.01$, and $p<0.005$, respectively.

rats were sacrificed by decapitation, the livers were perfused with ice-cold physiological saline $(0.9 \% \mathrm{NaCl})$, and then removed and homogenized with a 4 -fold volume of $1.15 \% \mathrm{KCl}$ solution. The cytosolic GSH-Px activities in liver homogenates were measured according to a method described by Paglia and Valentine $^{18}$ with some modifications. The cytosolic GSH-Px activities were standardized with protein concentrations, and expressed as $\mathrm{U} / \mathrm{mg}$ protein. The protein concentrations of the samples were measured using a Bio-Rad Protein Assay Kit (Bio-Rad Laboratories, Hercules, CA). The TBARS in liver homogenates were measured according to a method described by Ohkawa ${ }^{19}$ with some modifications, and expressed as nmol/g liver.

Aspartate aminotransferase (AST) and alanine aminotransferase (ALT)

Blood serum was obtained from $19 \mathrm{SeD}$ and 13 normal 8week-old rats. Assessments of the serum AST and the ALT levels were requested to Mitsubishi Kagaku Bio-Clinical Laboratories, Inc. (Tokyo, Japan).

\section{$P-450$ activity}

Five normal and $5 \mathrm{SeD}$ rats (6 weeks of age) were used for the P-450 assay. The rats were sacrificed by decapitation. The livers were perfused with ice-cold physiological saline $(0.9 \%$ $\mathrm{NaCl}$ ), then removed and homogenized with a 4-fold volume of a $1.15 \% \mathrm{KCl}$ solution. The $\mathrm{MC}$ fraction was obtained by ultracentrifugation. The microsomal P-450 activities were measured according to a method described by Omura and Sato ${ }^{20}$ with some modifications. The P-450 activities were standardized with protein concentrations and expressed as $\mathrm{nmol} / \mathrm{mg}$ protein. The protein concentrations of the samples were measured as described above.

\section{Results and Discussion}

Table 1 lists the contents of Se, Mn, Fe, Co, and $\mathrm{Zn}$ in the diet and the liver of rats, as measured by INAA. The Se contents in the $\mathrm{SeD}$ diet and the $\mathrm{SeD}$ rat liver were below the detectable level. The detection limit of Se was estimated to be 0.046 $\mathrm{mg} / \mathrm{kg}$ in the dried sample as the upper limit under the present INAA condition. The Mn contents in the SeD diet were 1/5 those in the normal diet, while the Mn contents in the liver were the same between the $\mathrm{SeD}$ and normal rats. The Fe contents in the SeD diet and the SeD rat liver were 1.2 fold and 2.5 fold 
Table 2 Cytosolic GSH-Px activities in normal and SeD rat liver homogenate

\begin{tabular}{lcc}
\hline & Normal rats & SeD rats \\
\hline Cytosolic GSH-Px [U/mg protein] & $376 \pm 272$ & $1.27 \pm 1.31$ \\
\hline
\end{tabular}

Five 8-week-old male Wistar rats were used. The values are shown as means \pm S.D. A significant difference was obtained between the normal and the SeD rats at $p<0.05$.

Table 3 Indices of hepatic damage of the normal and $\mathrm{SeD}$ male rats

\begin{tabular}{lll}
\hline & \multicolumn{1}{c}{ Normal rats } & \multicolumn{1}{c}{ SeD rats } \\
\hline AST [IU/L] & $76.2 \pm 11.6$ & $105.1 \pm 34.6^{*}$ \\
ALT [IU/L] & $29.1 \pm 5.8$ & $33.1 \pm 5.9$ \\
TBARS [nmol/g liver] & $301.8 \pm 115.5$ & $426.3 \pm 76.7$ \\
Liver weight [g] & $8.43 \pm 0.99$ & $5.31 \pm 0.78^{*}$ \\
Liver to body weight ratio [\%] & $3.86 \pm 0.40$ & $5.54 \pm 0.35^{*}$ \\
\hline
\end{tabular}

Eight-week-old male Wistar rats were used. AST and ALT were averaged in 19 rats for SeD group and 13 rats for normal group. TBARS was averaged in 5 rats for both group. The liver weight was averaged in 6 rats used for INAA analysis. Values are shown as means \pm S.D. $*$ indicates a significant difference obtained between the normal and the SeD rats at $p<0.005$.

greater than that in the normal diet and the normal rat liver, respectively. Although a small amount of blood remained in liver samples, the difference in blood $\mathrm{Fe}$ contents between the normal and SeD rats could be ignored. This is because of the following three reasons: hematocrit was at the same level between the normal and SeD rats; the hemoglobin amount was also the same; and the serum $\mathrm{Fe}$ contents were negligibly small. This finding confirms that $\mathrm{Fe}$ was accumulated in the SeD rat liver. The Co contents in the $\mathrm{SeD}$ diet and the $\mathrm{SeD}$ rat liver were $1 / 28$ and $1 / 20$ of those in the normal diet and normal rat liver, respectively. In addition, there were remarkable differences between the Co contents in the normal and the SeD diets. The Co contents in the liver showed a good correlation to those in the diet. Co is well-known as the active center of vitamin $B_{12}$. On the other hand, the role of inorganic Co is mostly unknown in animals. The normal and the SeD diets contained 0.034 and $0.017 \mathrm{mg} / \mathrm{kg}$ of additional vitamin $\mathrm{B}_{12}$, which are $2 / 3$ and $1 / 3$ of the recommended level in NRC-78, respectively. The actual contents of vitamin $B_{12}$ in both diets should be higher than those, because of the vitamin $B_{12}$ contents in natural materials for the diets. The level of vitamin $B_{12}$ in NRC-78 $(0.05 \mathrm{mg} / \mathrm{kg})$ corresponds to $0.0021 \mathrm{mg} / \mathrm{kg}$ of inorganic Co. It was demonstrated that the Co content in the $\mathrm{SeD}$ diet was sufficiently higher than the recommended level. No significant difference was observed in the $\mathrm{Zn}$ contents of either the diet or liver between the two groups.

The liver cytosolic GSH-Px activities are given in Table 2. The cytosolic GSH-Px activity in the liver homogenate of the normal rats was consistent with those reported for similar ages, ${ }^{21,22}$ while a markedly low cytosolic GSH-Px activity $(0.3 \%$ of the normal rats) was observed in the SeD rats. This confirms that the $\mathrm{SeD}$ rats in this experiment were exposed to severe $\mathrm{Se}$ deficiency, which caused an extreme malfunction of the cytosolic GSH-Px. A malfunction of the cytosolic GSH-Px increases the amount of hepatic endogenous $\mathrm{H}_{2} \mathrm{O}_{2}$ in bile ${ }^{23}$ and exposes the liver to severe oxidative stress caused by excess $\mathrm{H}_{2} \mathrm{O}_{2}$.
(A)

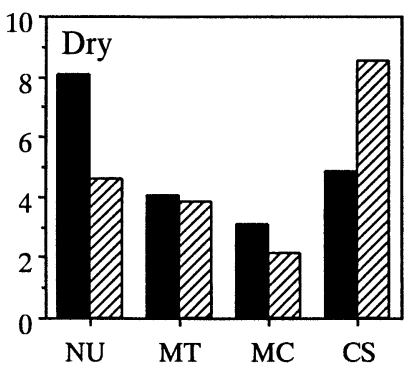

(B)

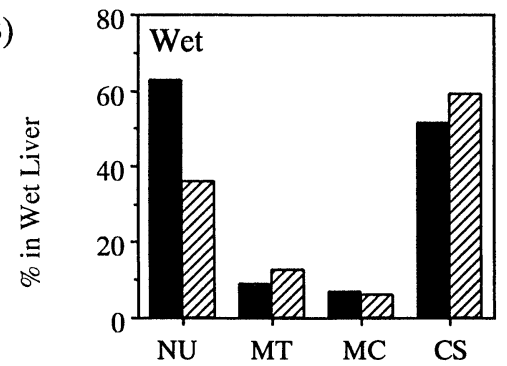

Fig. 1 Percentage of each cell fraction to the total wet liver weight of the normal rats (closed column) and the SeD rats (striped column). The values are shown as percentages of (A) the dry cell fraction samples for INAA and (B) the wet cell fraction samples for MTA to the wet liver weight. The wet weight of the CS fraction was corrected by the ratio of the total weight of homogenate to the total weight of liver.

Table 3 gives indices of the hepatic damage. The serum AST level was significantly higher in the SeD rats than in the normal rats. The serum ALT and the liver TBARS levels were also higher in the $\mathrm{SeD}$ rats than in the normal rats, although no significance was obtained. The percentage of liver weight to body weight was significantly larger in the $\mathrm{SeD}$ rats than in the normal rats. In a previous study by $\mathrm{Al}$-Bader et al. ${ }^{24}$ rats administered thioacetamide (which is known to cause Sedeficiency) developed liver cirrhosis and showed significant increases in both liver weight and the ratio of liver-to-body weight. In this study, the total amounts of each dry cell fraction for the INAA samples and each wet cell fraction for the MTA samples were weighed. The percentage of each fraction weight to the wet liver weight (g) was compared (Fig. 1). The weight of the NU fraction in the normal rat livers was greater than that in the livers of the SeD rats. Inversely, the CS fraction was greater in the $\mathrm{SeD}$ rats than in the normal rats. This fact suggests that the hepatocytes of the SeD rats had swollen, and that the liver had a hypertrophic inflammation. In this experiment, indices of hepatic damage, indicating a pathological condition in the liver of the SeD rats, were obtained along with the changes in the subcellular distribution of metal elements related to in vivo redox reactions.

The contents and uptake rates of $\mathrm{Se}, \mathrm{Mn}, \mathrm{Fe}, \mathrm{Co}$, and $\mathrm{Zn}$ in the liver were measured to estimate the details of the dynamics of these elements. Figure 2 shows the resulting content measurements (A, C, E, G, and I; left hand side of the figure) and the uptake rates (B, D, F, H, and $\mathrm{J}$; right hand side).

The Se contents in the normal rats obtained by INAA were $4.769 \pm 0.088 \mathrm{mg} / \mathrm{kg}$ in the NU, $3.796 \pm 0.267 \mathrm{mg} / \mathrm{kg}$ in the MT, $2.237 \pm 0.268 \mathrm{mg} / \mathrm{kg}$ in the MC, and $4.722 \pm 0.164 \mathrm{mg} / \mathrm{kg}$ in the CS fraction, respectively (Fig. 2A). In contrast, the Se contents in all of the separated fractions of the liver homogenate of the $\mathrm{SeD}$ rats were below the detection limit, which was estimated to be $0.046 \mathrm{mg} / \mathrm{kg}$ in the dried samples under the 
(A)

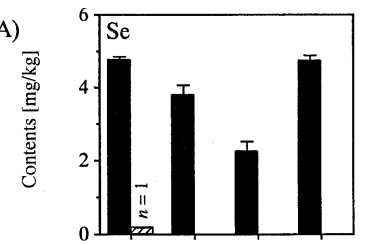

(C)

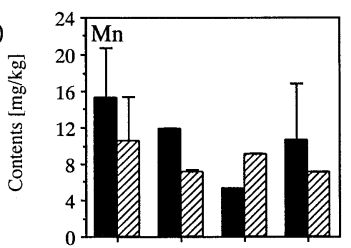

(E)

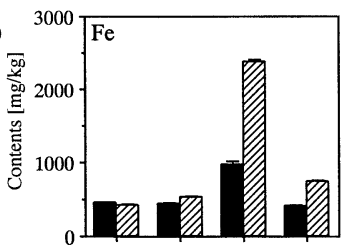

(G)

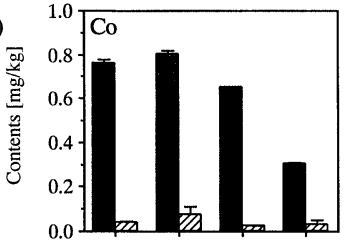

(I)

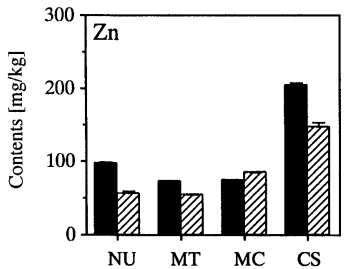

(B)

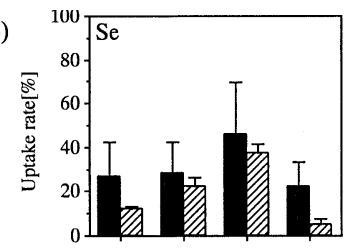

(D)

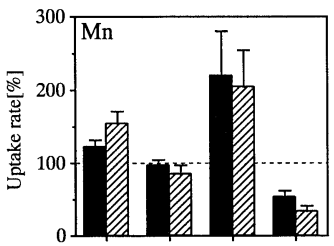

(F)

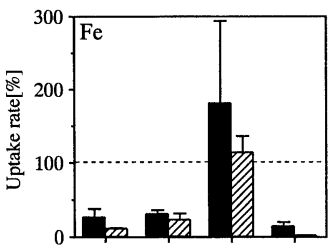

(H)

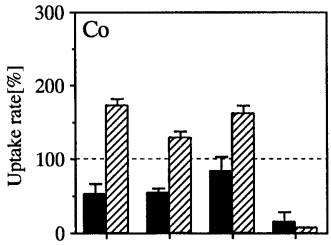

(J)

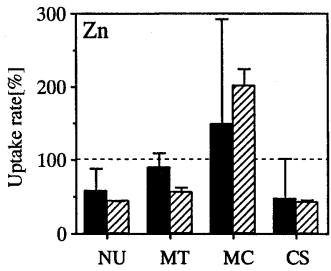

Fig. 2 Contents and uptake rates of Se, Mn, Fe, Co, and $\mathrm{Zn}$ in the liver cell fractions of the normal rats (closed column) and the SeD rats (striped column). Se contents (A) and uptake rates (B), Mn contents (C) and uptake rates (D), Fe contents (E) and uptake rates $(\mathrm{F})$, Co contents $(\mathrm{G})$ and uptake rates $(\mathrm{H}), \mathrm{Zn}$ contents $(\mathrm{I})$ and uptake rates $(\mathrm{J})$. The contents and uptake rates of these elements were measured with INAA and MTA, respectively.

present experimental conditions. The cytosolic GSH-Px activity was well correlated with the Se content in the liver, suggesting that there was a change of the redox valance in the SeD rats. There were no significant differences in the uptake rates of Se between the $\mathrm{SeD}$ and normal rats in any of the cell fractions (Fig. 2B). This may have been due to the absence of a specific transportation system for Se ions (for example, transferrin for Fe ions).

The Mn contents were lower in 3 fractions of the $\mathrm{SeD}$ rats (i.e., in all but the $\mathrm{MC}$ fraction) than in the corresponding fractions of the normal rats (Fig. 2C). Significant differences were observed in the MT fraction. However, no significant differences in Mn uptake rate were observed between the $\mathrm{SeD}$ and normal rats (Fig. 2D).

The Fe contents increased in the MC and the CS fractions of the $\mathrm{SeD}$ rats (Fig. 2E). The Fe contents in the $\mathrm{SeD}$ rats were $2377 \pm 26 \mathrm{mg} / \mathrm{kg}$ in the MC fraction, and $740 \pm 22 \mathrm{mg} / \mathrm{kg}$ in the $\mathrm{CS}$ fraction. These results agreed with those of a previous study in which $\mathrm{Fe}$ deposits were observed in lysosomes of the liver and kidney of SeD rats. ${ }^{6}$ Although the Fe uptake rate in the MC fraction of both the $\mathrm{SeD}$ and normal rats was higher than that in

Table 4 P-450 activities in normal and SeD rat liver microsomes

\begin{tabular}{ccc}
\hline & Normal rats & SeD rats \\
\hline P-450 [nmol/mg protein $]$ & $0.146 \pm 0.063$ & $0.325 \pm 0.135$ \\
\hline
\end{tabular}

Five 6-week-old male Wistar rats were used. The values are shown as means \pm S.D. No significant difference was obtained between the normal and $\mathrm{SeD}$ rats.

other fractions, no significant differences were observed between the $\mathrm{SeD}$ and normal rats under the experimental conditions (Fig. 2F). Since the activity of P-450 in microsomes of the SeD rat liver was higher than that in the normal rats (Table 4), the observed increase in the Fe contents of the MC fraction was probably due, at least in part, to the induction of P-450. On the other hand, if Fe had been present, there would likely have been an increase of hydroxyl radical synthesis through the Fenton reaction, leading to peroxidation of the lipid membrane. In fact, it has been reported that TBARS and other indices of lipid peroxidation increased in SeD animals..$^{25,26}$ Our previous finding that the uptake rate of $\mathrm{Fe}$ in the liver of $\mathrm{SeD}$ rats changed with time ${ }^{27}$ would seem to be in conflict with the present study, in which the Fe uptake did not change. Because the liver was not perfused in the previous case, the Fedistribution in residual blood might have affected the results to some extent. The iron uptake rate in the liver has been studied using rats overloaded with different amounts of Fe. ${ }^{7}$ The diquat-induced effects on the biliary concentrations of nonheme iron have also been observed. ${ }^{28}$ As a result of the redox stress, iron (e.g., ferritin or microsomal iron protein $)^{29,30}$ may have been released from intracellular binding pools. Se-deficiency may also have created an iron-overload in such pools, although we have not yet analyzed the chemical forms this iron may take.

The Co contents were also lower in $\mathrm{SeD}$ than in normal rats (Fig. 2G), which was attributed to the low content of Co in the $\mathrm{SeD}$ diet. In contrast, MTA data indicated that the uptake rate of Co increased in all cell fractions other than the CS fraction (Fig. $2 \mathrm{H}$ ), suggesting that the uptake rate was enhanced in order to fulfill the Co requirement in the $\mathrm{SeD}$ rat liver. The uptake rates in the NU, MT, and MC fractions of the SeD rats were higher than $100 \%$. This indicates that the uptake was larger than the uniform distribution of the tracer in the whole body. In a multitracer solution, Co exists in the form of inorganic ions. There was a significant difference in the uptake rate of inorganic Co between the normal and SeD rats, suggesting that inorganic Co may play an important role in in vivo redox system. Previous studies have reported that the levels of selenium in the livers and kidneys of $\mathrm{SeD}$ rats were positively related to dietary selenium and negatively related to an interaction between dietary selenium and cobalt. ${ }^{31,32}$ In accordance with these reports, the present results show that the contents of Se and Co in all of the cell fractions were influenced by the dietary contents, and also suggest a complementary effect between selenium and cobalt in the liver, although the present administrations of Se and Co were different than those used previously.

The $\mathrm{Zn}$ contents in the NU and CS fractions of the SeD rat livers were lower than those in the normal rats, but the MC fraction in the livers of SeD rats increased (Fig. 2I). Significant differences were observed in all of the fractions. However, no significant differences were observed in the $\mathrm{Zn}$ uptake rates in any fractions between the normal and SeD rats (Fig. 2J).

$\mathrm{Mn}$ and $\mathrm{Zn}$ are known as the active centers of Mn-SOD and 
$\mathrm{Cu} / \mathrm{Zn}$-SOD. Mn-SOD and $\mathrm{Cu} / \mathrm{Zn}-\mathrm{SOD}$ are located mainly in the MT and CS fractions, respectively. The SOD activity in these cell fractions should be measured in further study. Both increases and decreases of the SOD activities in various tissues of $\mathrm{SeD}$ rats have previously been reported. ${ }^{33,34}$ The relationships among $\mathrm{Se}, \mathrm{Zn}$, and $\mathrm{Mn}$ remain to be clarified.

Copper $(\mathrm{Cu})$ is usually determined by a radioactivity measurement of ${ }^{66} \mathrm{Cu}\left(T_{1 / 2}=5.1 \mathrm{~min}\right)$ in INAA. In this study, however, we did not focus our attention on such a short-lived nuclide. In addition, no suitable $\mathrm{Cu}$ radio isotopes were found in the MTA experiment for the present. The contents of $\mathrm{Cu}$ in sub-ppm amount of biological samples should be analyzed with ICP-MS and/or atomic absorption analysis. To estimate uptake rate of $\mathrm{Cu}$, it is necessary to use ${ }^{64} \mathrm{Cu}$ as single radio-tracer. An estimation of the $\mathrm{Cu}$ dynamics remains for a future experiment.

In this experiment, the normal diet had a relatively high nutritive value in almost all nutrients compared to the recommended level in NRC-78. The SeD diet had a lower nutritive value than the normal diet, except for some minerals (for example, $\mathrm{Fe}$ and $\mathrm{Zn}$ ), though these contents were almost sufficient for the recommended levels. There was a minor difference in the constituents of elements between the normal and SeD diets. Therefore, some ambiguities remain in a strict sense as to whether all of the results presented here originated only from Se-deficiency. For a more detailed estimation of the effect of Se-deficiency, Se should be added to the SeD diet to make a perfect control experiment. However, the present results demonstrate the possibility of a change in the distribution of metal ions caused by a low-Se diet.

In conclusion, the INAA results showed that the Fe content was highest in the MC fraction of both the normal and the $\mathrm{SeD}$ rats, and that the Fe content of the $\mathrm{SeD}$ rats increased to more than 2 fold that of the normal rats in the fraction. The contents of $\mathrm{Se}, \mathrm{Mn}, \mathrm{Co}$ and $\mathrm{Zn}$ were characteristic to each cell fraction. The MTA results showed that the highest uptake of $\mathrm{Se}, \mathrm{Mn}, \mathrm{Fe}$, and $\mathrm{Zn}$, but not of $\mathrm{Co}$, was found in the MC fraction, indicating that these metal ions were incorporated preferentially into the $\mathrm{MC}$ fraction. In addition, the Fe uptake rate was similar in each cell fraction between the $\mathrm{SeD}$ and the normal rats, and the uptake in the MC fraction was remarkably higher than that in the other 3 fractions. These results may suggest that the uptake rates of elements are not necessarily in agreement with the contents. To clarify the mechanisms of Fe uptake, the timedependent uptake rate should be estimated. Details on the chemical form and the mechanism for the transport of $\mathrm{Fe}$ and Co in a living body are in progress.

\section{Acknowledgements}

The authors thank Ms. Y. Ueda, H. Washimi, and H. Urata for their experimental help, and Drs. F. and S. Ambe of the Institute of Physical and Chemical Research (RIKEN) for their suggestion to use a multitracer. We are also grateful to the staff of RIKEN Ring Cyclotron for their help with the heavy-ion irradiation.

\section{References}

1. R. J. Magree and B. D. James, in "Handbook on Metals in Clinical and Analytical Chemistry", ed. H. G. Seiler, A. Sigel, and H. Sigel, 1994, Marcel Dekker, New York, $551-$ 562

2. R. F. Burk, Ann. Rev. Nutr., 1983, 3, 53 .
3. M.-E. Mirault, A. Tremblay, D. Furling, G. Trepanier, F. Dugre, J. Puymirat, and F. Pothier, Ann. New York Acad. Sci., 1994, 738, 104.

4. J. B. de Haan, F. Cristiano, R. C. Iamello, and I. Kola, Biochem. Mol. Biol. Int., 1995, 35, 1281.

5. N. Chareonpong-Kawamoto and K. Yasumoto, Biosci. Biotechnol. Biochem., 1995, 59, 302.

6. N. Chareonpong-Kawamoto, T. Higasa, and K. Yasumoto, Biosci. Biotechnol. Biochem., 1995, 59, 1913.

7. D. A. Panastasiou, D. V. Vayenas, A. Vassilopoulos, and M. Repanti, Pathol. Res. Practice, 2000, 196, 47.

8. Y. H. Lee, D. K. Layman, and R. R. Bell, J. Nutr., 1981, 111, 194.

9. P. M. Moriarty, M. F. Picciano, J. L. Beard, and C. C. Reddy, J. Nutr., 1995, 125, 293.

10. J. M. Matés, C. Pérez-Gómez, and I. N. de Castro, Clin. Biochem., 1999, 32, 595.

11. Y.-L. Huang, J.-Y. Sheu, and T.-H. Lin, Clin. Biochem., 1999, 32, 131.

12. J. Chaudiére and R. Ferrari-Iliou, Food Chem. Toxicol., 1999, 37, 949.

13. K. Matsumoto, K. Endo, and H. Utsumi, Biol. Pharm. Bull., 2000, 23, 641.

14. R. Hirunuma, K. Endo, M. Yanaga, S. Enomoto, S. Ambe, A. Tanaka, M. Tozawa, and F. Ambe, Appl. Radiat. Isot., 1997, 48, 727.

15. N. Sotogaku, K. Endo, R. Hirunuma, S. Enomoto, S. Ambe, and F. Ambe, J. Radioanal. Nucl. Chem., 1999, 239, 429.

16. D. G. Hafeman, R. A. Sunde, and W. G. Hoekstra, J. Nutr., 1974, 104, 580

17. R. Hirunuma, K. Endo, S. Enomoto, S. Ambe, and F. Ambe, Appl. Radiat. Isot., 1999, 50, 843.

18. D. E. Paglia and W. N. Valentine, J. Lab. Clin. Med., 1967, 70,158 .

19. H. Ohkawa, N. Ohishi, and K. Yagi, Anal. Biochem., 1979, 95, 351

20. T. Omura and R. Sato, J. Biol. Chem., 1964, 239, 2370.

21. L. E. Rikans, D. R. Moore, and C. D. Snowden, Biochim. Biophys. Acta, 1991, 1074, 195.

22. I. D. Capel and A. E. Smallwood, Res. Commun. Chem. Pathol. Pharmacol., 1983, 40, 367.

23. Y. Ueda, K. Matsumoto, and K. Endo, Biochem. Biophys. Res. Commun., 2000, 271, 699.

24. A. Al-Bader, H. Abul, T. Hussain, M. Al-Moosawi, T. C. Mathew, and H. Dashti, Mol. Cell. Biochem., 1998, 185, 1.

25. S. S. Sobajic, M. B. Mihailovic, and M. O. Miric, J. Environ. Pathol. Toxicol. Oncol., 1998, 17, 265.

26. R. W. Scholz, L. A. Minicucci, and C. C. Reddy, Biochem. Mol. Ciol. Int., 1997, 42, 997.

27. R. Hirunuma, N. Sotogaku, K. Endo, S. Enomoto, S. Ambe, and F. Ambe, J. Radioanal. Nucl. Chem., 1999, 239 , 213.

28. S. Gupta, L. K. Rogers, and C. V. Smith, Toxicol. Appl. Pharmacol., 1994, 125, 42.

29. D. W. Reif, Free Rad. Biol. Med., 1992, 12, 417.

30. G. Minotti, Chem. Res. Toxicol., 1993, 6, 134.

31. M. R. Gardiner and H. Nicol, Aust. J. Exp. Biol. Med. Sci., 1971, 49, 291.

32. J. B. Van Ryssen, W. J. Miller, R. P. Gentry, and M. W. Neathery, J. Dairy Sci., 1987, 70, 639.

33. L. L. Ji, F. W. Stratman, and H. A. Lardy, J. Am. Coll. Nutr., 1992, 11, 79.

34. K. Asayama, N. W. Kooy, and I. M. Burr, J. Lab. Clin. Med., 1986, 107, 459. 